\title{
"Ich glaube, es ist eine Illusion, dass wir die Menschheit zur Zahnseide bringen"
}

Ende letzten Jahres fand in Köln eine Expertenrunde zur Studie „Klinische Wirksamkeit und Akzeptanz von Philips Sonicare AirFloss Ultra auf die approximale Gesundheit bei Erwachsenen“ statt (I. Ensmann, Uniklinik Köln). Daran nahmen teil: der Leiter der Studie Prof. Dr. Michael Noack (Direktor der Poliklinik für Zahnerhaltung und Parodontologie, Klinikum der Universität Köln), StudienMitautor Prof. Dr. Stefan Zimmer (Lehrstuhl für Zahnerhaltung und Präventive Zahnmedizin, Universität Witten/Herdecke) und Sylvia Fresmann (Dentalhygienikerin, Vorsitzende der Deutschen Gesellschaft für DentalhygienikerInnen DGDH e.V.), Dr. Klaus Höcker (Facharzt für Parodontologie, Vorsitzender des Berufsverbandes der Fachzahnärzte und
Spezialisten für Parodontologie BFSP e.V.) und PD. Dr. Dr. h.c. Adrian Kasaj (Oberarzt an der Poliklinik für Zahnerhaltungskunde, Universität Mainz). Im Expertengespräch in Köln fasste Studienleiter Noack die Bedeutung der klinischen Studie für die Praxis zusammen. Er ist überzeugt: „Mit den Ergebnissen eröffnen sich ganz neue Möglichkeiten, eben gerade weil man nicht mehr darüber diskutieren muss, welches Mittel das Beste ist, um approximal plaquefrei zu sein." Stattdessen könne man die verschiedenen Optionen aufführen. „Patienten, die Zahnseide gern und richtig anwenden, würde ich nicht umstimmen wollen“, betonte Noack. „Ich glaube aber, es ist eine Illusion, dass wir die Menschheit zur Zahnseide bringen. Denn umge-

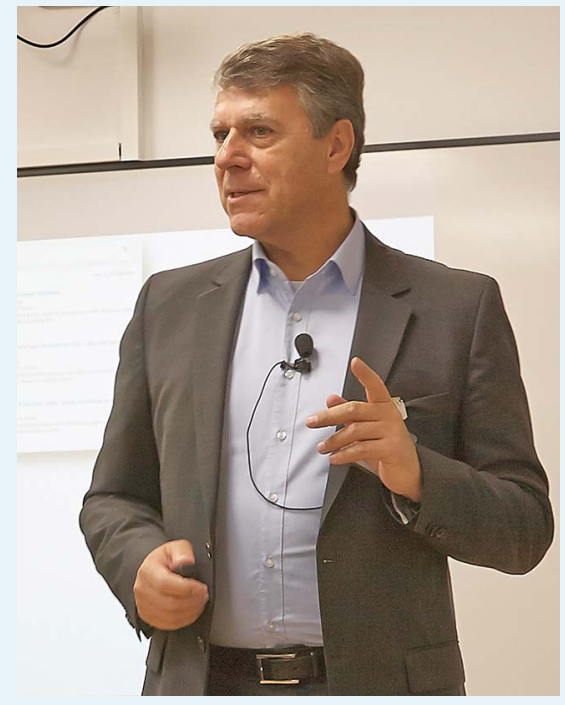

kehrt gibt es eben auch diejenigen, die mit einem technisch hochwertigen Hilfsmittel wie dem AirFloss besser umgehen können.“

Nach einer Pressemitteilung der Philips GmbH, Hamburg www.philips.de/sonicare 